\title{
Designing Local Food Service Ecosystem for Sustainability: An Agent-based Social Simulation Approach based on Service-Dominant Logic
}

\author{
Viet-Cuong Trieu \\ Institute of Service Science \\ National Tsing Hua University \\ cuongtv@iss.nthu.edu.tw
}

\author{
Fu-ren Lin \\ Institute of Service Science \\ National Tsing Hua University \\ frlin@iss.nthu.edu.tw
}

\begin{abstract}
This paper aims to design a local food service ecosystem based on the Service-Dominant Logic to overcome the limitation of the alternative food networks in terms of sustainability. The three core components of this service ecosystem are trust mechanism, cooperative model, and blockchain-based service platform. The service design approach and agent-based social simulation method are used to design and evaluate the service ecosystem. From the simulation results, an actor-to-actor network trust mechanism and an intelligent cooperative model are proposed based on evaluating sustainability in economic, social, and environmental aspects. This study's results contribute to service design methodology and practical service ecosystem development for sustainability.
\end{abstract}

\section{Introduction}

The concern about the unsustainability of conventional food systems leads to the growth in various "alternative" forms of food production and distribution, which is called "alternative food networks" (AFNs). AFNs is an umbrella term that includes a variety of distribution and production practices, such as farmers' markets, community supported agriculture (CSA), farm shops, consumer food cooperatives, and others [1]. AFNs seek to diversify and transform modern food provisioning by connecting ethical producers and customers in more local, direct ways [2]. While there is some empirical evidence supporting the potential sustainability impacts of AFNs, there are also a high number of studies suggesting that the impacts may not be so straightforward. In terms of economic sustainability, many studies show that selling directly to customers can bring higher profit but requires more resources and time, so the net profits may not live up to the theory [3]. Environmental critics claim that local produce's food mileage may be shorter, but the carbon footprint will not be lower than the conventional one because of farmers' inefficient transportation [4]. In terms of social impact, AFNs have overlooked low- income customers [5] and does not preserve the food culture as expected [6].

In Taiwan, the most popular form of AFNs is the farmers' market. The first farmers market, named Chi Mei, arose in 2006 in Kaohsiung City, then many farmers' markets started to appear in the following years [7]. However, at present, almost all farmers' markets in Taiwan only exist on a small scale. From an economic, social, and environmental perspective, the farmers market model in Taiwan also encounters limitations, as mentioned in the criticisms of AFNs. This creates the motivation for this study to design a local food service ecosystem to overcome these limitations. Moreover, a local food network that can enhance the interaction between farmers and customers has an essential meaning in Taiwan's context. Currently, about $80 \%$ of Taiwanese live in cities, and this proportion continues to increase while the overall population tends to decrease. Taiwan is facing the problem of urban-rural disconnection. Therefore, a local food service ecosystem can contribute to urban-rural sustainability.

The local food service ecosystem is designed in the context of Taiwan based on four meta-theoretical foundations of the Service-Dominant Logic (S-DL): actor-to-actor (A2A) networks, resource liquefaction, resource density, and resource integration [8]. The local food network is designed as an A2A network in which actors co-create value through resource integration. A combination of trust mechanism, intelligent co-op support system, and blockchain-based service platform can enhance resource density and leverage resource liquefaction through information sharing. An agentbased social simulation method is used to evaluate and optimize the designed system. Using the design practice and agent-based social simulation approach based on SDL, this study theoretically contributes to the service design methodology. This study is the first research using a simulation approach to evaluate two opposite trust mechanism approaches (positive reinforcementHITS versus negative reinforcement- TSM) in an actorto-actor (A2A) network. Finally, the research results contribute to the development of a practical service ecosystem for sustainability in Taiwan. 


\section{Background}

\subsection{The sustainability promises of AFNs}

Different from conventional food systems, AFNs has three core characteristics: increased requirement for product and production, reduced distance between producer and customer, and new forms of market governance [1]. The products circulating in AFNs are often characterized as fresh, natural, organic, quality, and slow, while the production is described as environmentally benign using traditional production methods [2]. The distance between producers and customers is characterized by localness, the small size of networks, transparency, information, and "shortening" the supply chain. AFNs also generate new forms of food markets or 'new ways to coordinate production, purchasing, and commercialization' such as CSA, farmer market, customer or producer cooperatives .

The AFNs' core characteristics contribute to sustainability in three aspects: economic, social, and environmental. The environmentally friendly production choices can positively impact all aspects of environmental sustainability [9]. The reduced physical distance in AFNs is believed to contribute to environmental sustainability by reducing the transportation distances of foods or "food mileage," equaling less fuel use and $\mathrm{CO}_{2}$ emissions [10].

In terms of economic sustainability, AFNs contribute to producer livelihoods and employment creation by adding value through differentiated production methods and reduced informational distance, enabling them to receive a higher price from the market. Social embeddedness brings customers to accept higher prices due to the nature of the exchange [11]. The reduction of value chain distance allows a greater share of value captured by the producer [12]. Indeed, bypassing middlemen and selling through direct markets may be the only way of getting access to markets for some small producers [13]. The new forms of governance in AFNs are also thought to contribute to producer livelihoods. Arrangements such as CSA are built on the idea of sharing the economic risk in agriculture between producers and customers or producer co-operatives, including collectively having more negotiating power and resources, thus better market possibilities and income. Finally, thinking beyond individual producers or workers, the reduced physical distance in AFNs means money spent on food contributes to the local economy.

In the social sustainability aspect, properties of food and its production and reduced physical distance contribute to customer health. Natural foods are believed to be healthier than highly processed foods.
Many activists and customers believe that the reduced physical distance in AFNs means food is fresher and retains more nutrients than food transported over long distances [14]. For example, organic farming restricts the use of chemicals or antibiotics in production, thus addressing product and producer safety and health impacts and greater biodiversity. Locality and diversity of production are considered critical for food security [10], and reduced value chain distance can also improve access to foods [13]. AFNs characteristics are also thought to have positive effects on food culture. The focus on territorial embeddedness and traditional production methods is argued to preserve regional, traditional food cultures, and diversity [10]. Food culture can also be supported by increased visibility and awareness of food provenance and production, which stems from the reduced informational distance in AFNs.

AFNs may also create indirect sustainability impacts related to learning and participation. Reduced informational distance is widely thought to increase participants' learning and awareness of sustainabilityrelated issues in the food system. Increased learning and awareness, in turn, are believed to lead to more sustainable practices [15]. These indirect impacts can be understood as feeding into and reinforcing participant values and the choices about preferred production methods, the form and length of food supply chains, and governance arrangements.

\subsection{The criticism on AFNs}

Although AFNs promise to make a significant contribution to sustainability, it also faces many criticisms. In terms of economic sustainability, reduced value chain distance raises the question of how value is being redistributed? While there is case study evidence supporting the prominent argument that reduced value chain distance results in producers capturing a greater share of the value [16], producers' net benefit has been questioned. Many studies suggest that direct selling to customers may require more resources, time, and energy from the producers, and its profitability, in the end, may not live up to the theory [3]. For example, from an empirical study on the farmers market, James [17] raised a question: Have farmers received enough income from farmers' markets to cut ties with mainstream retail outlets? If a lack of customers, farmers may suffer losses, including financial loss and an accompanying loss of working time on the farm. Agricultural jobs in rural areas that already lack the labor force will be more and more shortage if farmers spend more time on the farmers market. Therefore, the farmers could not economically sustain attendance at the market, and farmers' markets cannot provide a viable alternative for small-scale producers. 
In the environmental impact aspect, reduced food mileage may not be as significant as the mode of transport in reducing transport-related emissions [4]. Transportation also generally causes only a small part of the life-cycle greenhouse gas emissions of food [18]. The environmental impacts of food production also differ in different localities with different development conditions [2, 14]. Also, Small-scale local food distribution may be inefficient with the conventional transportation model [4]. In the social influence aspect, reducing physical distance would mean that food is fresher and more nutritious has been challenged by considering the time, not just distance in transport [14]. The codification linked to, for instance, geographic indication labels may promote standardization rather than protecting the diversity of traditional production and, thus, preservation of food culture [6]. "Valueadded" products may benefit the producer but be out of the reach of lower-income customers [5]. Indeed, there seems to be tension between farmer livelihoods' goals and access to affordable food [13].

\section{Design the local food service ecosystem}

Under the influence of rapid urbanization, Taiwan is facing the problems of rural decline and urban-rural disconnection. Farmers' market, the most common form of AFNs in Taiwan, also encounters limitations, as mentioned in the criticisms of AFNs. In this study, we design an local food service ecosystem to overcome criticisms of AFNs (Table 1) based on four metatheoretical foundations of SD-L: actor-to-actor (A2A) networks, resource liquefaction, resource density, and resource integration $[19,20]$.

The A2A network can be formed from traditional local food networks, rural tourism, and cultural festival activities. The service platform is designed to support A2A network formation so that anyone who participates in a local food network, rural tourism, or cultural festival can become an actor. Information sharing (viewed as resource liquefaction) between farmers and customers can mitigate the food loss and waste cost. The service platform can enhance resource density by supporting searching, mixing, matching, and integrating resources [19]. The service platform also needs to be designed to integrate all traditional and digital resources in the most effective way to co-create value and support actors to perform their roles to co-create value.

The proposed service ecosystem's foundation is the decentralized blockchain-based service platform, which has three components: blockchain technology, trust mechanism, and co-op model. Blockchain technology mentioned in this study is the third-generation blockchain, a combination of different techniques to enable blockchain-based applications, including cryptography methods, peer-to-peer networks, digital signatures, and distributed ledger technology, smart contract technology, consensus mechanism, among others.

Table 1. Criticism on AFNs and system design

Criticism on AFNs $\quad$ Service ecosystem design

\begin{tabular}{l|l}
\hline Economic: AFNs are & A Blockchain-based service
\end{tabular}

inefficient, consume a lot of platform will minimize the costs resources, time, not as as incurred in the current AFNs beneficial as expected. models.

Social: Unclear in protecting Trust and transparency ensure the diversity of traditional healthy products. A lower price production and food culture due to the lower cost will better preservation; not ensure the support lower-income

high quality and healthy customers. The service platform product; not support low- maintains interaction in the A2A income customers because network and contributes to food of a higher price. culture preservation.

Environment: "Food Collaboration and information mileage" may not reduce sharing help optimize because small-scale local transportation and increase local food distribution may be product consumption, leading to inefficient in shipping. $\quad$ reduced food mileage.

Compared with the centralized platform, a blockchain-based platform has more economic advantage by reducing transaction and technology costs. A blockchain-based platform ensures data transparency, transaction traceability, along with the self-executable smart contract that triggers transactions under the predefined condition, will significantly reduce transaction costs. Blockchain technology can also reduce technology cost related to platform maintenance and data protection through the consensus and verify mechanism, which guarantees the immutability and transparency of the transactions, prevent the network failure and fraud, thus, generating the trust via the exchange protocol without any need for a central regulator or a trustable third-party [21].

The local food service ecosystem needs to be designed to provide quality products by establishing trust between customers and producers. Although Blockchain technology can provide trust through data transparency and integrity, it does not guarantee actors' trustworthiness in the A2A network. Therefore, a trust mechanism, which can determine actor trustworthiness from the $\mathrm{A} 2 \mathrm{~A}$ network-level perspective, will play a crucial role in the online service platform.

The third component of the service platform is the "co-op model" that supports value co-creation based on information sharing on the trusted A2A network. The proposed co-op model can operate without a lead actor's involvement with the support of the Intelligent Co-op Decision Support System (ICDSS). The ICDSS, which supports decision-making based on the defined rule of exchange, combined with the trust mechanism, data 
transparency, and information sharing, can replace the traditional co-op model manager role. This mechanism allows actors flexibly to collaborate in the A2A network without a lead actor's role, hence significant savings in management costs.

With the features mentioned above, the designed service ecosystem can overcome the criticisms of AFNs. The service ecosystem supports direct exchange between farmers and customers at a lower cost and minimizes the food loss and waste cost, which is estimated by $30 \%$ of the food produced [22]. In terms of social effect, low-income customers will have more opportunities to access healthy local agricultural products, and more actors participating in A2A Network will contribute to food culture preservation. In the environmental aspect, collaboration and information sharing help optimize food distribution, somewhat reduce food mileage. Also, an increase in local produce consumption will lead to a decrease in agricultural products transported from other locations, contributing to reducing food mileage.

\subsection{Propose trust mechanism in A2A Network}

We propose the "trust mechanism" to compute each actor's trustworthiness based on the interaction between actors within the network. An actor's trust score is determined through two steps: (1) compute inter-actor trust based on the interaction between actors, and (2) compute the actor trust score from a network-level perspective using the social network trust algorithm.

3.1.1 Inter-actor trust computation. The inter-actor trust between a trustor and trustee is determined based on the trust model of Meyer et al. [23]. The degree of inter-actor trust is a function of the trustee's perceived Ability (A), Benevolence (B), and Integrity (I). In an A2A food network, the customer plays a trustor role while the producer plays a trustee role. The trustee's ability is measured by the quality of the product. Benevolence is determined by how much effort the producer fulfills the needs of the customer. Integrity is defined as the producer's promise not to cancel the order once agreed to fulfill the order. In Equation 1, $\boldsymbol{r}$ is the discount rate determining the degree of decay of interactor trust overtime. Once the inter-actor trust is reevaluated, the lastest trust evaluation has a higher weight of influence.

$$
\operatorname{Trust}_{A 2 A}=\sum_{i=0}^{n}\left(A_{i}+B_{i}+I_{i}\right) * r^{t_{n}-t_{i}}(1)
$$

3.1.2. Social network trust algorithm. In recent social network trust research, a node's trust score is determined by a pair of complementary scores: trustingness $(\mathrm{ti})$ and trustworthiness $(t w)$. Trustingness of an actor is defined as his or her propensity to trust others (trustor role). A higher trustingness score implies that the actor has a high propensity to trust others in the network. Trustworthiness refers to how trustworthy an actor is perceived by others (trustee role). A higher trustworthiness score means the actor is a highly trustworthy person in the network.

3.1.3. HITS and TSM algorithm. To compute actors' trust scores from a network-level perspective, we offer two opposite approaches, the positive reinforcement approach- HITS versus the negative reinforcement approach- TSM, then use the simulation method to choose the appropriate algorithm. HITS algorithm, which initially is web pages ranking algorithm, is recently used to compute trust scores in social networks in some researches [24, 25], while TSM is a new algorithm proposed by Roy et al. [26] in 2017.

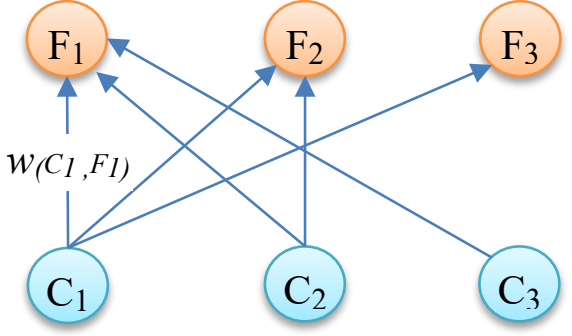

Figure 1 Simple example of A2A network

In the field of social network trust research, the trustingness score of a node is computed based on the "out link" weight (inter-actor trust) and trustworthiness score of all nodes which it connects to, and the trustworthiness of a node is computed based on the "in link" weight and trustingness score of all nodes which it connects from. For instance, as shown in Figure 1, the trustingness of customer node $\left(\mathrm{C}_{1}\right)$ is computed from the trustworthiness of all farmer node $\left(\mathrm{F}_{1-3}\right)$ and "out link" weight from $\mathrm{C}_{1}$ to other farmer nodes. The remarkable difference between HITS and TSM is the "positive" or "negative" reinforcement approach.

\begin{tabular}{|c|}
\hline HITS Algorithm \\
\hline $\begin{array}{l}t i(c)=\sum_{\forall x \text { in out }(c)} t w(x) * w(c, x)(2) \\
t w(f)=\sum_{\forall x \text { in }(f)}^{t i(x) * w(x, f)}\end{array}$ \\
\hline TSM Algorithm \\
\hline $\begin{array}{l}t i(c)=\sum_{\forall x \text { in out }(c)} \frac{w(c, x)}{1+t w(x)} \\
t w(f)=\sum_{\forall x \text { in }(f)} \frac{w(x, f)}{1+t i(x)}\end{array}$ \\
\hline
\end{tabular}

HITS algorithm (as shown in Equations 2 and 3) enforces a positive reinforcing relation between trustingness and trustworthiness score (i.e., increase one measure of a node leads to an increase in the other 
measure of its neighbors). HITS hypothesizes that a high trustor will trust a high trustee so that a higher trustingnesss score of trustor leads to its neighbor's higher trustworthiness score. On the contrary, TSM hypothesizes that a higher trustingnesss score contributes to its neighbor's trustworthiness a lower degree. TSM algorithm (Equations 4 and 5) enforces a negative reinforcing relation, the increase of trustingness a node leads to a decrease of the trustworthiness of other nodes it links to. As the example in Figure 1, in HITS, $\mathrm{C}_{1}$ contributes a higher degree to the $F_{1}$ trustworthiness score; in TSM, $C_{1}$ contributes a lower degree to the $\mathrm{F}_{1}$ trustworthiness.

\subsection{Intelligent cooperative food model}

Unlike the conventional food model based on competition, the cooperative food system emphasizes actors' cooperation for mutual benefits. In the past decade, many studies and practices have shown that implementing the local food co-op model benefits both farmers and customers. Two critical issues of the local food coop model that need to be addressed are "trust and transparency" (members do not trust each other because of lack of information transparency) and "the co-op management costs". This study proposed an intelligent cooperative food model based on blockchain technology to solve these two issues.

Trust and information transparency: the blockchain-based platform supports food traceability and information transparency so that that trust can be achieved based on the proposed trust mechanism and information transparency.

The co-op management costs: the management costs can be minimized thanks to the Intelligent Co-op Decision Support System (ICDSS), which supports the matching between supply and demand based on supply capacity and trust score in a fairway. The ICDSS can replace the role of the co-op management board. The co-op model is operated based on the principle of collaboration and information sharing (Figure 2) include the following steps:

(1) The farmer shares the product supply capacity in the determined period before harvesting.

(2) Customers share their future demands (e.g., issue an order one week ahead).

(3) Co-op members (farmer, subscribed customer) vote to determine the selling price.

(4) The ICDSS distributes customer orders to farmers based on the supply capacity and trustworthiness score of farmers with the principle: a greater trust score and a higher supply capacity will receive more orders (see the simulation section for details).

(5) Farmers harvest agricultural products, aggregate and transport them to their customers.

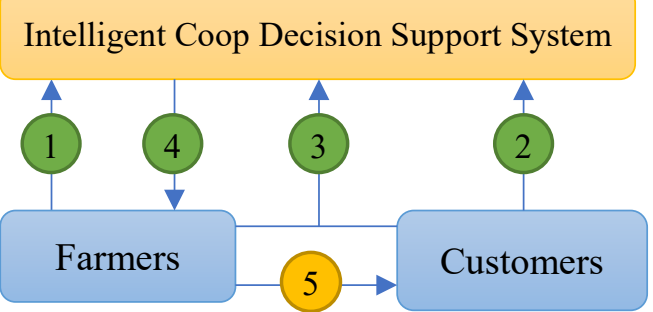

Figure 2 The intelligent coop model

In this model, customers collaborate by sharing demand information earlier, and farmers collaborate by sharing their supply ability before they start to harvest. Based on the shared information, the system optimizes the matching between supply and demand. It then provides recommendations to support farmers in deciding when and how much quantity to be harvested.

\section{Agent-based social simulation model}

The agent-based social simulation model in this study was developed based on the mesa framework [27]. The simulation method is used to evaluate the proposed local food service ecosystem by answering two questions:

(1) Which trust algorithm is more appropriate for the designed service ecosystem: HITS or TSM?

(2) What is the difference between the co-op model and the non-coop model from the economic, social, and environmental aspects of sustainability?

The simulation model is built based on the context of Hsinchu, a city in the north of Taiwan, and its surrounding rural area. The data used in this simulation was constructed from field data and the statistics bureau. In Hsinchu area, more than $50 \%$ of farmers own less than 0.5 ha farm size [28]. There are two types of farms in the Hsinchu area: mountain farms whose main products are vegetables and flat-land farms that grow rice. Farmers in this model are small-scale farmers, so the farm size is set to less than 0.5 ha for mountain farms and less than one ha for flat-land farms. We assume that mountain farms grow cabbage, corn, and carrot while the flat-land farms grow rice. The simulation model is composed of three types of agents: farmers, city households, and intelligent co-op agents, in which farmers and households are human agents, and the intelligent co-op agent is a machine agent representing the ICDSS.

\subsection{The increase of household agents over time}

At present, in Hsinchu, small farmers' agricultural products are sold mainly to the conventional market. We assume that, in the beginning, only 30 farmers and 30 households participate the service ecosystem. Over 
time, the number of households will increase until demand reaches farmer supply capacity. We use the Bass Diffusion Model [29] to simulate this growth. The market size $(M=7000)$ is estimated from $5 \%$ of the total households in Hsinchu city [28]. The new household agent in each period is computed monthly using Equation 6, in which the coefficient of innovation $(p=0.001)$ and the coefficient of imitation $(q=0.04)$ is set based on the study of Massiani and Gohs [30].

$$
N(t)=M p+(q-p) A(t)-\frac{q}{M} A(t)^{2}
$$

\subsection{Simulation process}

The overall process of the local food simulation model in a single time-step is illustrated in Figure 3. Once the simulation model starts, farmers will randomly select the start time of cultivation (weeks 1-14 for vegetables and weeks 8-11 for rice). Vegetables are grown year-round and will be replanted 1-2 weeks after harvest, except carrots can only be grown from December to April. Rice will be planted two crops a year, the first crop in February and the second crop in July. Once planted, the farm status changes to the sowing period within 9 to 16 weeks, depending on the type of product. The harvest time of vegetables can happen for several weeks, while rice is harvested all at once. When it comes to harvest time, farmers will decide when and how much to harvest. For vegetables, in the non-coop model, farmers harvest the same quantity in several weeks, while in the coop model, the farmer will decide how much to harvest based on the received orders from the intelligent co-op agent.

In the non-coop model, The household agent will decide to buy from which farmer based on the product price and farmer trustworthiness. In the coop model, an intelligent coop agent allocates orders to farmers based on the decision-making rule (presented in section 4.3). In the "product trading" step, customers will evaluate farmers' trustworthiness based on service quality. Service quality is defined as product quality, delivery time, information, and instructions related to the product. This rating will be updated to the "inter-actor trust" of the A2A network. After all transactions are done, the trust algorithm (HITS or TSM) computes trustingness and trustworthiness scores throughout the entire network. The service platform is assumed to be deployed from year two onwards. Therefore, in the first year, all harvested products will be sold to conventional markets at wholesale prices. From the $2^{\text {nd }}$ year onwards, farmers will sell their products through the designed service platform, and the rest will sell to conventional markets. Vegetable products must be sold all during the harvesting week, while rice can be stocked and only need to be sold all until the harvest time of the next crop.

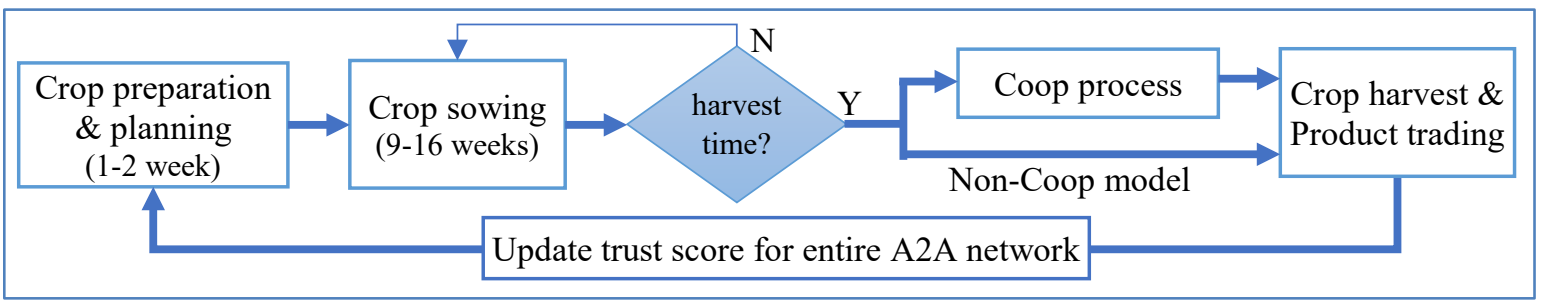

Figure 3 Flowchart of the overall local food system simulation model in singe time-step

\subsection{Decision-making rule}

4.3.1. The non-coop model. Household agents in the model are assumed to make decisions based on the preference ranking score of farmers. The score calculation (Equations 7) is the Cobb-Douglas functional form of customer utility and the farmer's trustworthiness score adapted to the proposed function by Klos and Nooteboom [31]. Because the customer has different price sensitivity; therefore, $\beta$ of each customer will be randomly selected within $[0.1,0.9]$.

$$
\begin{gathered}
\text { Score }_{i}=(\text { Customer Utility })^{\beta_{i}} \cdot(\text { Farmer Trust })^{1-\beta_{i}} \text { (7) } \\
\text { Customer Utility }=\frac{1-e^{-\left(P_{\max }-P\right) / R}}{1-e^{-\left(P_{\max }-P_{\min }\right) / R}}
\end{gathered}
$$

The farmer's trustworthiness score is computed by the trust algorithm (HITS or TSM). The customer utility value (Equations 8) is expressed in an exponential utility function scaled in the range from zero to one. $P_{\min }$ is the wholesale price and $P_{\max }$ is the highest retail price, which is estimated at $150 \%$ of the wholesale price. It is assumed that customers are risk-averse in which the utility function is concave [32] so that the risk-tolerant $\mathrm{R}$ is set to $P_{\min }$ for all household agents. The selling price $\mathrm{P}$ is decided by the farmer. At the initial stage, the farmer agent sets the $\mathrm{P}$ to $0.99 P_{\max }$. Farmers will consider to decrease product price at $5 \%, 10 \%$, or not if they cannot sell anything through the service platform for two consecutive weeks. 
4.3.2. The co-op model. In the co-op model, farmer agents will update their supply capacities before harvesting, and household agents will share their demands one week ahead by issuing orders earlier. The system will accept orders until it reaches the maximum supply capacity. The intelligent co-op agent then assigns the order to farmers based on the proposed farmer ranking score (Equation 9). After one order is assigned, the farmer ranking score is recalculated. The decision rule in Equation 9 ensures a reasonable allocation of orders base on the quantity sold and the farmer's trustworthiness score. $\beta$ is set to 0.1 to increase the influence of the trust score. Farmers with a higher trustworthiness score or greater supply capacity will have a higher priority in receiving orders. However, once a farmer receives orders from the system, this farmer's priority will reduce, and other farmers will be given higher priority to receive orders.

$$
\text { Score }_{i}=\left(1-\frac{\text { Qty sold }}{\text { Qty capacity }}\right)^{\beta_{i}} \cdot(\text { Farmer Trust })^{1-\beta_{i}}(9)
$$

\section{Experimentation and results}

\subsection{Experimental setup}

The product characteristics are set in Table 2 . There are 30 farmer agents in which 15 farmers own 0.8 ha of flat-land farms, and 15 farmers own 0.25 ha of mountain farms. In terms of service quality, farmer agents are divided into three groups of 10 farmers each. The high trust group always provides highquality service. The ordinary trust group provides regular quality service, and the low trust group provides both regular and low quality of service with a $50 \%$ probability.

Table 2 Parameters setup for the experimentation

\begin{tabular}{|l|l|l|l|l|}
\hline \multicolumn{1}{|r|}{ Produces parameters } & Carrot & Cabbage & Corn & Rice \\
\hline Harvest time (weeks) & $10-12$ & $11-16$ & $9-14$ & $15-16$ \\
\hline Product yield (ton/ha) & 10 & 40 & 9 & 2 \\
\hline Wholesale price (USD/kg) & $\$ 1.2$ & $\$ 0.5$ & $\$ 2.5$ & $\$ 4$ \\
\hline Farm size allocation (\%) & $30 \%$ & $20 \%$ & $50 \%$ & $100 \%$ \\
\hline $\begin{array}{l}\text { Household demand } \\
\text { (kg/week) }\end{array}$ & $0-7$ & $0-10$ & $0-6$ & $0-2$ \\
\hline
\end{tabular}

The number of the household agent is 30 in the first week, and it increases over time. The household agents are divided into three groups: rational group, socially desirable group, and no-rating group. Household agents in the rational group are supposed to rate 1 for high-quality service, [0.6-0.8] for regularquality, and [0-0.3] for low-quality. The socially desirable household agents have social desirability bias so that they tend to rate in a way that they believe to be viewed favorably by farmers. Therefore, they will rate 1 for both high and regular quality and [0.4$0.5]$ for the low-quality service. The socially desirable group is set due to the simulation context is set in Taiwan. Because of cultural differences, individuals from Eastern societies are likely to exhibit more socially desirable responses than Western societies [33]. Finally, in the end, the no-rating group was assumed not to rate the service quality. In case the quality was too low, they will rate it at $[0-0.3]$.

\subsection{Analysis of results}

\subsubsection{Trust algorithms evaluation: HITS and TSM}

Before the "negative reinforcement" approach in the TSM algorithm was first proposed in 2017, all social network trust algorithms are "positive reinforcement" approaches [26]. This study explores the difference between these two approaches by comparing the HITS and TSM algorithms. The simulation period can be divided into three stages: initial, growth, and maturity. The initial stage (week 152 ) is the period before deploying the service ecosystem. The growth stage begins from week 53, in which the local trading volume increases rapidly due to the product demand is less than supply. Finally, after three years, when product demand nearly reaches the supply capacity, it reaches the maturity stage.
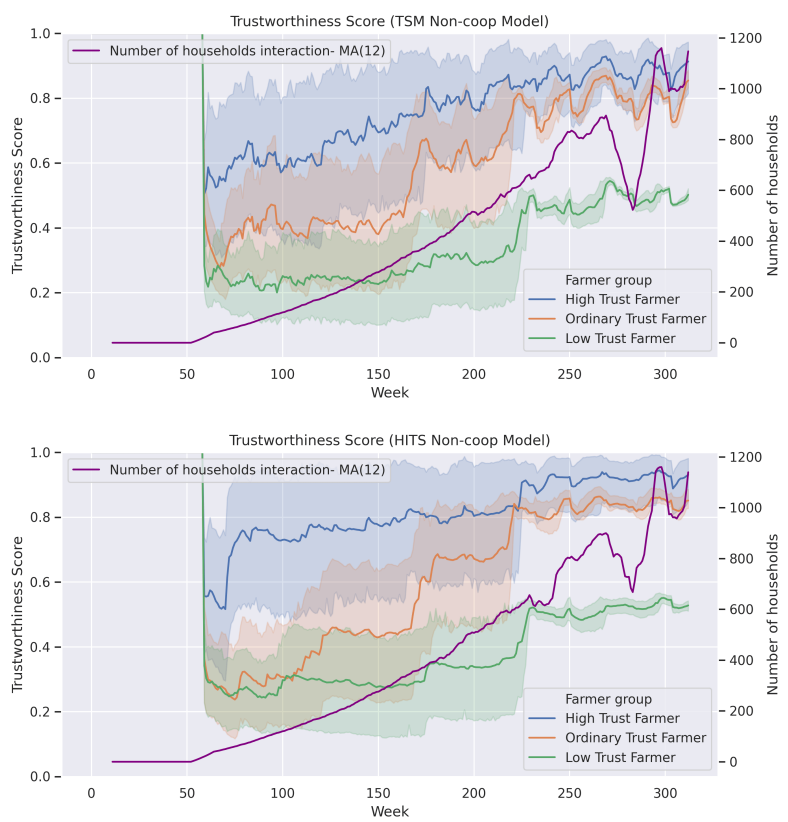

Figure 4. Farmer trustworthiness in the Non-coop model

The farmers' trustworthiness score is observed over time along with the increase in the number of household agents under different scenarios. From the results of many simulations, we found that, in the scenario that the number of household agents in the 
"rational group" is higher than other groups combined, both HITS and TSM can separate three farmers groups well. However, HITS starts to produce worse results in case there are more socially desirable household agents. We set up the simulation scenario in which the household agents proportion in the three groups are as follows: rational group (40\%), socially desirable group (40\%), no-rating group (20\%). As shown in Figure 4, the TSM separates very well the three group farmers, while HITS gives a worse result. We dug deeper to explain this phenomenon and found that HITS will have a problem in a system with many high ratings due to the bias. In HITS, the trustingness and trustworthiness score, number of links, and link weight are all positive reinforcement. Therefore, when the socially desirable agent rates the highest score for high or regular quality service, they will get a higher trustingness score and significantly increase the farmer's trustworthiness. This can make the farmer's trustworthiness change abnormally during some periods.
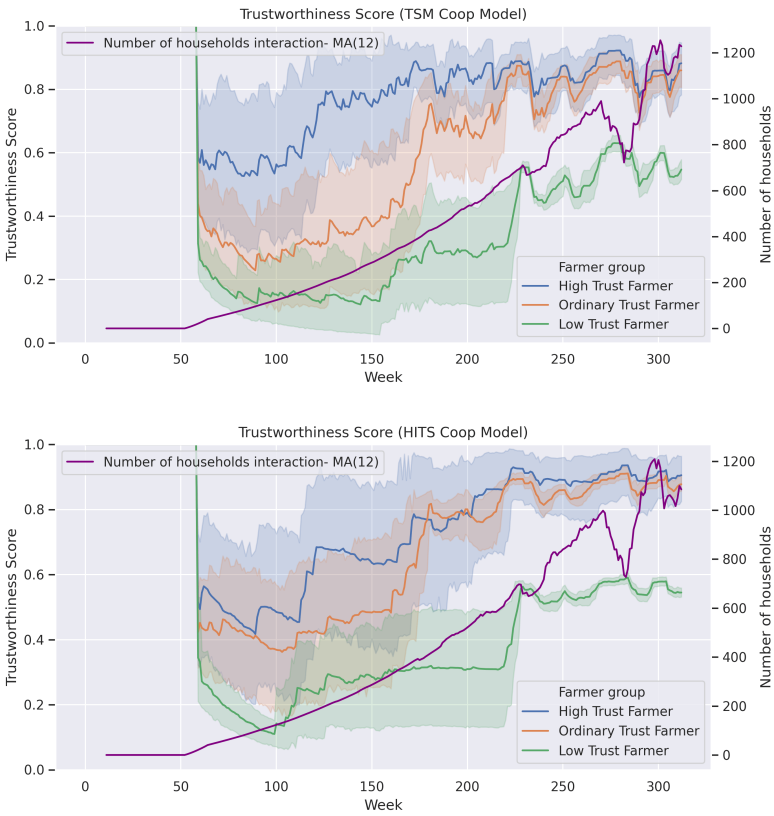

Figure 5. Farmer trustworthiness in the Coop model

We perform another experiment with the same scenarios for the co-op model. As shown in Figure 5, TSM still produces better results than HITS. To explore the TSM algorithm's disadvantages, we tested several scenarios and found that TSM is very sensitive to low ratings. In situations that household agents are dishonest and intentionally evaluate the high trust farmer at the lowest grade. The impact of this low rating will be amplified many times and significantly affect the farmer's trustworthiness score. However, these low ratings are outline samples, which can easily be excluded. Because the relationship between customers and local farmers tend to increase over time in Asian culture, social desirability bias is inevitable. Therefore, we identify that the TSM algorithm is more appropriate than HITS for our designed local food service ecosystem.

\subsubsection{Sustainability impact evaluation}

This section compares the economic, social, and environmental impact between the non-coop versus co-op model using the TSM algorithm.
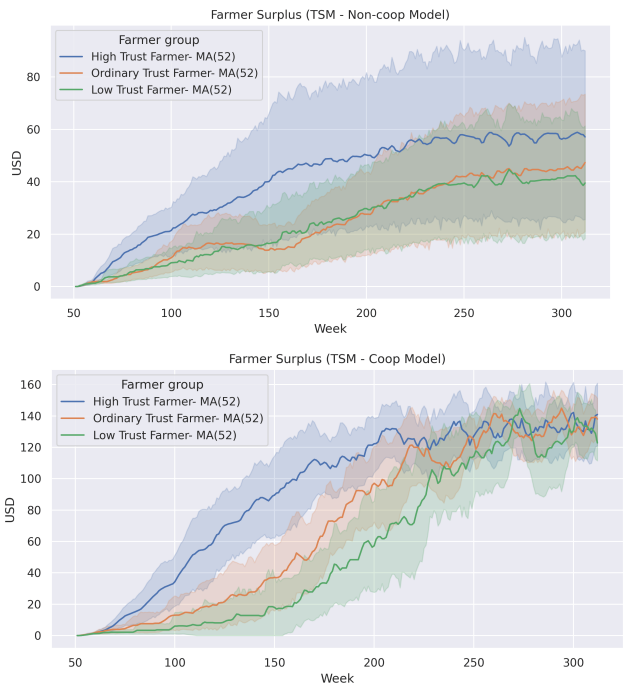

Figure 6. Farmer surplus in non-coop \& coop model

Economic impact: In terms of economic impact, both the non-coop and co-op models bring more benefits to farmers than AFNs, such as farmer markets, because they are implemented on blockchainbased low-cost systems. The differences between these two models are evaluated by comparing farmer surplus. Farmer surplus is defined as the additional profit farmer received by selling through the service ecosystem than the conventional market. As shown in Figure 6, in the non-coop model, the high trust farmer group gets the highest surplus due to receiving more orders while there was no significant difference in surplus between the ordinary and low trust farmer groups due to these groups tend to decrease their product prices to get more orders. In the co-op model, the surplus of all farmer groups is significantly higher than in the non-coop model because there is no price competition, and farmers can sell more through the service platform thanks to the optimization in harvesting. In the co-op model, the surplus is also more equitably divided between different farmer groups, and the surplus variance within a group is also smaller compared to the non-coop model. To get a higher surplus, farmers will try to improve the quality of their products and services rather than reducing product prices. 
Social impact: We measure social influence based on the number of farmer interactions with city households. The higher the number of interactions (or more local product is exchanged through service platform), the more people in the city will be able to get healthy food, increase awareness of product production, and preserve food culture. Increasing and sustaining interaction also contributes to urban-rural sustainability. As shown in Figure 7, the number of interactions in the co-op model is slightly higher than the non-coop model. In the long term, the co-op model will bring a higher surplus to farmers, and farmers also have sufficient information on customer demand. Therefore, they will have an incentive to expand production, increasing the number of interactions.
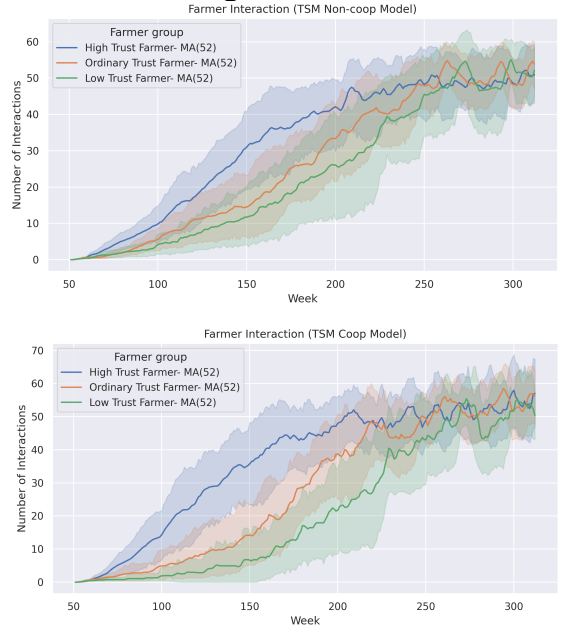

Figure 7. Farmer interaction in Non-coop \& Coop model
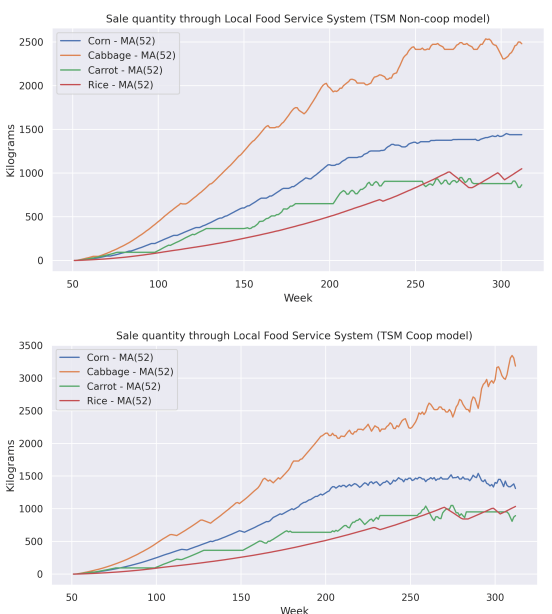

Figure 8. Sale quantity in Non-coop \& Coop model

Environment impact: We assess the environmental impact based on "food mileage." In Hsinchu City, local products will travel around 20 to $40 \mathrm{~km}$ from the rural area compared to 200 to $300 \mathrm{~km}$ from a large-scale farm in southern Taiwan. As shown in Figure 8, although the number of products sold through the service ecosystem in the co-op model is only slightly higher than the non-coop model, however, in the co-op model, farmers can easily aggregate products and optimize transportation to reduce costs and "food mileage" due to the collaboration and information sharing. Moreover, food loss and waste cost in the traditional supply chain is estimated at around $30 \%$ of the food produced [22]; therefore, the more local product is exchanged through the service platform, the less product from other locations (south of Taiwan and global) is consumed lead to the reduction of food mileage.

\section{Discussion}

In this study, we propose a service ecosystem based on blockchain technology, trust mechanism, and coop model, and then use the simulation method to evaluate the trust mechanism and co-op model. The simulation results show that the TSM trust mechanism is more appropriate in Taiwan context. We also propose an intelligent co-op model without a leading actor in which the product exchanges are allocated based on the farmers' trust score, sold quantity, and supply capacity. However, our decision-making rule is still a bit simple and only supports farmers in harvesting decisions. In future studies, this rule needs to be improved to allocate benefits equitably and reasonably among co-op members. The intelligent coop model can be developed to support farmers' collaboration and decision-making regarding product selection, cultivation time, and land size. The simulation model can be developed to serve as a tool for scenario-based strategic planning.

\section{Conclusion}

This study designed a local food service ecosystem for sustainability based on four metatheoretical foundations of Service-Dominant Logic to overcome the limitations of the current form of AFNs. The agent-based social simulation method is used to evaluate and optimize the designed service ecosystem. The simulation results show that the co-op model, combined with the TSM trust mechanism, obtains the best results. This study's result is significant and could serve as a prerequisite for implementing the practical service ecosystem for sustainability in Taiwan. In terms of implementation, the designed service ecosystem can be deployed based on blockchain technology to ensure data transparency and minimize operating costs. An intelligent co-op system will optimize resource integration, increase resource density, and support farmers' decision-making. 


\section{Acknowledgment}

This research is sponsored by the Taiwan Ministry of Science and Technology (MOST) Grant \#1092410-H-007-019-MY2.

\section{References}

[1] S. Forssell and L. Lankoski, "The sustainability promise of alternative food networks: an examination through "alternative" characteristics," Agriculture and human values, vol. 32, no. 1, pp. 63-75, 2015.

[2] F. Edwards, "Alternative food networks," Encyclopedia of food and agricultural ethics, pp. 1-7, 2016.

[3] R. Milestad, L. Westberg, U. Geber, and J. Björklund, "Enhancing adaptive capacity in food systems: learning at farmers' markets in Sweden," Ecology and Society, vol. 15, no. 3, 2010.

[4] D. Coley, M. Howard, and M. Winter, "Local food, food miles and carbon emissions: A comparison of farm shop and mass distribution approaches," Food policy, vol. 34, no. 2, pp. 150$155,2009$.

[5] D. Goodman, E. M. DuPuis, and M. K. Goodman, Alternative food networks: Knowledge, practice, and politics. Routledge, 2012.

[6] S. Bowen and K. De Master, "New rural livelihoods or museums of production? Quality food initiatives in practice," Journal of Rural Studies, vol. 27, no. 1, pp. 73-82, 2011.

[7] F. R. Lin and S. Y. Wang, "Service Value Network Formation for Organic Agricultural Produce: An Actor Network Theory Perspective," in 2014 47th Hawaii International Conference on System Sciences, 2014: IEEE, pp. 1296-1305.

[8] S. L. Vargo and R. F. Lusch, "It's all B2B... and beyond: Toward a systems perspective of the market," Industrial marketing management, vol. 40, no. 2, pp. 181-187, 2011.

[9] T. Gomiero, D. Pimentel, and M. G. Paoletti, "Environmental impact of different agricultural management practices: conventional vs. organic agriculture," Critical reviews in plant sciences, vol. 30, no. 1-2, pp. 95-124, 2011.

[10] M. Schönhart, M. Penker, and E. Schmid, "Sustainable local food production and consumption: challenges for implementation and research," Outlook on agriculture, vol. 38, no. 2, pp. 175-182, 2009.

[11] R. Lee, "Shelter from the storm? Geographies of regard in the worlds of horticultural consumption and production," Geoforum, vol. 31, no. 2, pp. 137-157, 2000.

[12] T. Marsden, J. Banks, and G. Bristow, "Food supply chain approaches: exploring their role in rural development," Sociologia ruralis, vol. 40, no. 4, pp. 424-438, 2000.

[13] J. Guthman, A. W. Morris, and P. Allen, "Squaring farm security and food security in two types of alternative food institutions," Rural sociology, vol. 71, no. 4, pp. 662-684, 2006.

[14] B. Born and M. Purcell, "Avoiding the local trap: Scale and food systems in planning research," Journal of planning education and research, vol. 26, no. 2, pp. 195-207, 2006.

[15] Å. Sundkvist, R. Milestad, and A. Jansson, "On the importance of tightening feedback loops for sustainable development of food systems," Food policy, vol. 30, no. 2, pp. 224-239, 2005.
[16] J. Kirwan, "Alternative strategies in the UK agro-food system: interrogating the alterity of farmers' markets," Sociologia ruralis, vol. 44, no. 4, pp. 395-415, 2004.

[17] S. W. James, "Beyond' local' food: how supermarkets and consumer choice affect the economic viability of small-scale family farms in Sydney, Australia," area, vol. 48, no. 1, pp. 103-110, 2016.

[18] Y. Virtanen et al., "Carbon footprint of food-approaches from national input-output statistics and a LCA of a food portion," Journal of Cleaner Production, vol. 19, no. 16, pp. 1849-1856, 2011.

[19] R. F. Lusch and S. Nambisan, "Service innovation: A servicedominant logic perspective," MIS quarterly, vol. 39, no. 1, 2015.

[20] S. L. Vargo and R. F. Lusch, "Evolving to a new dominant logic for marketing," Journal of Marketing, no. 68, pp. 1-17, 2004.

[21] J. Pereira, M. M. Tavalaei, and H. Ozalp, "Blockchain-based platforms: Decentralized infrastructures and its boundary conditions," Technological Forecasting and Social Change, vol. 146, pp. 94-102, 2019.

[22] M. Rezaei and B. Liu, "Food loss and waste in the food supply chain," International Nut and Dried Fruit Council, pp. 26-27, 2017.

[23] R. C. Mayer, J. H. Davis, and F. D. Schoorman, "An integrative model of organizational trust," Academy of management review, vol. 20, no. 3, pp. 709-734, 1995.

[24] H. Cui, Q. Li, H. Li, and Z. Yan, "Healthcare fraud detection based on trustworthiness of doctors," in IEEE Trustcom/BigDataSE/ISPA, 2016: IEEE, pp. 74-81.

[25] L. Fernandez-Luque, R. Karlsen, and G. B. Melton, "HealthTrust: trust-based retrieval of youtube's diabetes channels," in Proceedings of the 20th ACM international conference on Information and knowledge management, 2011, pp. 1917-1920.

[26] A. Roy, J. Huh, A. Pfeuffer, and J. Srivastava, "Development of Trust Scores in Social Media (TSM) algorithm and application to advertising practice and research," Journal of Advertising, vol. 46, no. 2, pp. 269-282, 2017.

[27] D. Masad and J. Kazil, "MESA: an agent-based modeling framework," in 14th PYTHON in Science Conference, 2015, pp. 53-60.

[28] Taiwan National Statistics, "2015 Agriculture, Forestry, Fishery and Animal Husbandry Census in

Taiwan," 2015. [Online]. Available: https://eng.stat.gov.tw/.

[29] F. M. Bass, "Comments on "a new product growth for model consumer durables the bass model"," Management science, vol. 50, no. 12_supplement, pp. 1833-1840, 2004.

[30] J. Massiani and A. Gohs, "The choice of Bass model coefficients to forecast diffusion for innovative products: An empirical investigation for new automotive technologies," Research in Transportation Economics, vol. 50, pp. 17-28, 2015.

[31] T. B. Klos and B. Nooteboom, "Agent-based computational transaction cost economics," Journal of Economic Dynamics and Control, vol. 25, no. 3-4, pp. 503-526, 2001.

[32] P. R. Garvey, Analytical methods for risk management: A systems engineering perspective. Crc Press, 2008.

[33] K. L. Middleton and J. L. Jones, "Socially desirable response sets: The impact of country culture," Psychology \& Marketing, vol. 17, no. 2, pp. 149-163, 2000. 